\title{
SURFACES OF LEAST AREA WITH PARTIALLY FREE BOUNDARY ON A MANIFOLD SATISFYING THE CHORD-ARC CONDITION ${ }^{1}$
}

\author{
BY JOHANNES C. C. NITSCHE
}

Communicated by M. H. Protter, May 21, 1970

Consider a configuration in Euclidean 3-space consisting of a surface $T$ and of a rectifiable Jordan arc $\Gamma=\{\mathfrak{x}=z(\tau) ; 0 \leqq \tau \leqq 1\}$ having its end points on $T$, but no other point in common with $T$. Denote by $P$ the semidisc in the $(u, v)$-plane $P=\left\{u, v ; u^{2}+v^{2}<1, v>0\right\}$, by $\partial^{\prime} P$ and $\partial^{\prime \prime} P$ its boundary portions $\left\{u, v ; u^{2}+v^{2}=1, v>0\right\}$ and $\{u, v ;-1<u<1, v=0\}$, respectively, and by $P^{\prime}$ the domain $P \cup \partial^{\prime} P$.

A surface $S=\left\{\mathfrak{x}=\mathfrak{x}(u, v) ;(u, v) \in P^{\prime}\right\}$ is said to be bounded by the above configuration, or chain $\langle\Gamma, T\rangle$, if its position vector $\mathfrak{x}(u, v)$ $=\{x(u, v), y(u, v), z(u, v)\}$ satisfies the following conditions:

(i) $\mathfrak{x}(u, v) \in C^{0}\left(P^{\prime}\right)$.

(ii) $\mathfrak{x}(u, v)$ maps the arc $\partial^{\prime} P$ onto the open $\operatorname{arc}(\Gamma)=\{\mathfrak{x}=\{(\tau)$; $0<\tau<1\}$ monotonically in such a way that

$$
\lim _{\theta \rightarrow+0} \mathfrak{x}(\cos \theta, \sin \theta)=\mathfrak{z}(0), \quad \lim _{\theta \rightarrow \pi-0} \mathfrak{x}(\cos \theta, \sin \theta)=\mathfrak{z}(1) .
$$

(iii) The relation $\lim _{n \rightarrow \infty} d_{T}\left[\mathfrak{r}\left(u_{n}, v_{n}\right)\right]=0$ holds for every sequence of points $\left(u_{n}, v_{n}\right)$ in $P^{\prime}$ converging to a point on $\overline{\partial^{\prime \prime} P}$.

Here $d_{T}[\mathfrak{x}]=\inf _{\mathrm{t} \in T}|\mathfrak{x}-\mathrm{t}|$ denotes the distance between the point $\mathfrak{x}$ and the surface $T$.

Obviously, the convergence specified under (iii) is uniform in the following sense:

$$
\lim _{\delta \rightarrow 0} \sup _{(u, v) \in P^{\prime} ; 0<\text { os } \delta} d_{T}[\mathfrak{x}(u, v)]=0 .
$$

Thus while the distance function $d_{T}[\mathfrak{x}(u, v)]$ is continuous in $\bar{P}$, the same cannot generally be said about the vector $x(u, v)$. In fact, the trace of $S$ on $T$, i.e. the set of limit points on $T$ for all sequences $\mathfrak{x}\left(u_{n}, v_{n}\right)$ as in (iii) above, may well look quite bizarre. Examples illustrating such contingencies can be found in [2, pp. 95-96] and [4, pp. 220-222].

AMS 1970 subject classifications. Primary 49F10, 53A10; Secondary 26A63, 35B45, 49F25.

Key words and phrases. Surfaces of least area, minimal surfaces, surfaces minimizing Dirichlet's integral, free boundary value problem, least area property, free boundary, trace, regularity of free boundary, chord-arc condition, length-area principle, lift-off condition.

1 This work was supported by Grant number AFOSR 883-67. 
Denote by $\mathfrak{u}$ the collection of surfaces $S=\left\{\mathfrak{x}=\mathfrak{x}(u, v) ;(u, v) \in P^{\prime}\right\}$ which are bounded by the chain $\langle\Gamma, T\rangle$ and whose position vector belongs to the regularity class $C^{0}\left(P^{\prime}\right) \cap H_{2}^{1}(P)$. Here $H_{2}^{1}(P)$ is the pertinent Sobolev space insuring that $\mathfrak{x}(u, v)$ possesses square summable generalized derivatives. If the end points of $\Gamma$ can be connected on $T$ by a rectifiable arc, then the resulting closed contour spans a surface of class $\mathfrak{U}$. In $1938 \mathrm{R}$. Courant proved that, whenever the class $\mathfrak{U}$ is not empty, there exists in $\mathfrak{U}$ a surface $S=\{\mathfrak{x}=\mathfrak{x}(u, v)$; $\left.(u, v) \in P^{\prime}\right\}$ minimizing the value of Dirichlet's integral

$$
D_{P}[\mathfrak{x}]=\frac{1}{2} \iint_{P}\left(\mathfrak{x}_{u}^{2}+\mathfrak{x}_{v}^{2}\right) d u d v ;
$$

see $[1] ;[2$, pp. 87-96]; [4, pp. 201-223], and for further results [5], [6], and [15]. The position vector of the solution surface has the following additional properties:

(ii') The mapping of $\partial^{\prime} P$ onto $(\Gamma)$ is topological.

(iv) $\mathfrak{x}(u, v)$ is harmonic in $P$ and satisfies in $P$ the conditions

$$
\mathfrak{x}_{u}^{2}=\mathfrak{x}_{v}^{2}, \quad \mathfrak{x}_{u} \mathfrak{x}_{v}=0 .
$$

For the last three decades it has been a problem of great challenge to study the regularity of the solution surface on its free boundary and the nature of its trace. In this context the search for minimal conditions on the bounding surface $T$ which would guarantee the continuity of the trace deserves particular attention. Over the years the problem has been investigated for the case of planes [4], [15], closed orientable analytic surfaces [10] and, "admissible" surfaces of class $C^{m}, m \geqq 2,[8],[14]$ as bounding surfaces. Further remarks and results are contained in [3], [7]. It is the purpose of this paper to report a number of new theorems concerning the trace for the case of more general bounding surfaces- $C^{1}$-surfaces and surfaces satisfying a CA (=chord-arc) condition.

The proofs rest on the "least area property" of the solution surface which never seems to have been formulated or proved before, although the corresponding, but easier to demonstrate, property of the classical solution for Plateau's problem is well documented.

THEOREM 1. The area of a surface of class $\mathfrak{U}$ whose position vector minimizes Dirichlet's integral (in this class) simultaneously represents the minimum of the (Lebesgue) areas of all surfaces bounded by the chain $\langle\Gamma, T\rangle$.

The proof of Theorem 1 relies on facts from the theory of Lebesgue 
area and on devices due to H. Lebesgue [9, pp. 386-388 and p. 388], E. J. McShane [11, pp. 722-724] and R. Courant [2, pp. 89-91] and $[4$, pp. 203-205].

For reasons of convenience also the complex notation will be used: Setting $w=u+i v=\rho e^{i \theta}$ we shall interchangeably write $\mathfrak{x}(u, v)$, or $\mathfrak{x}(w)$, or $\mathfrak{x}(\rho, \theta)$-whichever is most expedient.

The surface $T$ is said to satisfy a CA-condition with constant $c_{1}>0$ if there is a positive number $\eta$ such that any two points $t_{1}$ and $\mathrm{t}_{2}$ of $T$ of distance $\left|\mathrm{t}_{2}-\mathrm{t}_{1}\right|$ less than $\eta$ can be connected on $T$ by an arc whose length is smaller than $\left(1+c_{1}\right)\left|t_{2}-t_{1}\right|$. If $T$ is a differential geometric regular closed (i.e. compact) orientable surface of class $C^{1}$, imbedded in space, then $T$ satisfies a CA-condition with every constant $c_{1}>0$. Also many unbounded surfaces have this property.

THEOREM 2. If the bounding surface $T$ satisfies a $C A$-condition with constant $c_{1}$, then the solution vector $\mathfrak{x}(u, v)$ (minimizing Dirichlet's integral in the class $\mathfrak{U}$ ) has a continuous extension to the closure of each domain $P_{a}=\left\{u, v ; u^{2}+v^{2}<a^{2}, v>0\right\}, 0<a<1$, belonging to class $C^{0, \beta_{1}}\left(\overline{P_{a}}\right)$. Here $\beta_{1}=2\left(2+c_{1}\right)^{-2}$.

THEOREM $2^{\prime}$. If $T$ is a surface of class $C^{1}$ as above, then the solution vector $\mathfrak{x}(u, v)$ has a continuous extension to each domain $P_{a}, 0<a<1$, belonging to class $C^{0, \beta}\left(P_{a}\right)$. Here $\beta$ is an arbitrary number in the interval $0<\beta<\frac{1}{2}$.

The demonstration of Theorem 2 is based on the length-area principle in conjunction with Theorem 1 and a kind of sewing theorem involving the construction of a surface of bounded area with specific properties. In view of a well-known lemma of C. B. Morrey [12, pp. 134-135], the inequality

$$
D\left[\mathfrak{x} ; w_{0}, \rho\right]=\frac{1}{2} \iint_{B\left(w_{0 ; \rho)}\right.}\left(\mathfrak{x}_{u}^{2}+\mathfrak{x}_{v}^{2}\right) d u d v \leqq M_{\rho}^{2 \beta_{1}}
$$

where $B\left(w_{0} ; \rho\right)=\left\{w ; w \in P,\left|w-w_{0}\right|<\rho\right\}$, is crucial. For $w_{0}=u_{0}$ $+i v_{0} \in \bar{P}_{a}$ only the case $0<v_{0}<\rho<\delta$ requires an elaborate proof. Set $\Phi(r)=D\left[\mathfrak{x} ; u_{0}, r\right]$. For almost all $r$ in $v_{0}<r<v_{0}+\delta$ the following is true:

(1) There are no branch points of $S$ on the arc $\Lambda_{r}=\left\{w=u_{0}+r e^{i \theta}\right.$; $\Phi<\theta<\pi\}$, i.e. $\mathfrak{x}_{\theta}\left(w_{0}+e^{i \theta}\right) \neq \mathrm{p}$ for $0<\theta<\pi$.

(2) The derivative $\phi^{\prime}(r)$ exists and

$$
\Phi^{\prime}(r)=\int_{0}^{\pi}\left|\mathfrak{x}_{r}\left(w_{0}+r e^{i \theta}\right)\right|^{2} r d \theta .
$$


Choose $\delta, 0<\delta<(1-a) / 2$, so that

$$
\frac{1}{2} \iint_{|w|<1 ; 0<v<2 \delta}\left(\mathfrak{x}_{u}^{2}+\underline{\mathfrak{x}}_{v}^{2}\right) d u d v<\frac{1}{\pi}\left(\frac{2+c_{1}}{2}\right)^{2} \eta^{2} .
$$

We now assert that $2 \beta_{1} \Phi(r) \leqq r \Phi^{\prime}(r)$ for almost all $r$ in $v_{0}<r<v_{0}+\delta$. Assume that $r \Phi^{\prime}(r)<2 \beta_{1} \Phi(r)$ for such a value. Then the limits

$$
\mathfrak{x}^{\prime}=\lim _{\theta \rightarrow+0} \mathfrak{x}\left(u_{0}+r e^{i \theta}\right) \text { and } \mathfrak{x}^{\prime \prime}=\lim _{\theta \rightarrow \pi \rightarrow 0} \mathfrak{x}\left(u_{0}+r e^{i \theta}\right)
$$

exist. $\mathfrak{x}^{\prime}$ and $\mathfrak{x}^{\prime \prime}$ are points on $T$ and

$$
\begin{aligned}
\left|\mathfrak{x}^{\prime \prime}-\mathfrak{x}^{\prime}\right|^{2} \leqq l^{2}(r) & =\left(\int_{0}^{\pi}\left|\mathfrak{x}_{\theta}\left(u_{0}+r e^{i \theta}\right)\right| d \theta\right)^{2} \leqq \pi \int_{0}^{\pi} \mathfrak{x}_{\theta}^{2}\left(u_{0}+r e^{i \theta}\right) d \theta \\
& \leqq \pi r \int_{0}^{\pi} \mathfrak{x}_{r}^{2}\left(u_{0}+r e^{i \theta}\right) r d \theta=\pi r \Phi^{\prime}(r)<2 \pi \beta_{1} \Phi(r)<\eta^{2}
\end{aligned}
$$

Thus the points $\mathfrak{x}^{\prime}$ and $\mathfrak{x}^{\prime \prime}$ can be joined on $T$ by an arc $\Lambda_{2}$ whose length is smaller than $\left(1+c_{1}\right) l(r)$. The closed contour consisting of the arcs $\Lambda_{r}$ and $\Lambda_{2}$ bounds a disc-type surface $\Sigma$ of area

$$
A(\Sigma)<\frac{1}{4 \pi}\left[l(r)+\left(1+c_{1}\right) l(r)\right]^{2} .
$$

If $\mathfrak{x}^{\prime} \neq \mathfrak{x}^{\prime \prime}$ this surface can be given a representation $\sum=\{\mathfrak{x}=\mathfrak{y}(w)$; $\left.w \in \overline{B\left(u_{0} ; r\right)}\right\}$. Here the vector $\mathfrak{y}(w)$ is continuous in $\overline{B\left(u_{0} ; r\right)}$ and analytic in $B\left(u_{0} ; r\right)$. Moreover-and this is the essential part$\mathfrak{y}\left(u_{0}+r e^{i \theta}\right)=\mathfrak{r}\left(u_{0}+r e^{i \theta}\right)$ for $0<\theta<\pi$. In view of the minimizing property of the surface $S$ an application of Theorem 1 now leads to

$$
D\left[\mathfrak{x} ; u_{0}, r\right] \leqq A(\Sigma)<\frac{\left(2+c_{1}\right)^{2}}{4 \pi} l^{2}(r) \leqq\left(\frac{2+c_{1}}{2}\right)^{2} r \Phi^{\prime}(r)=\frac{1}{2 \beta_{1}} r \Phi^{\prime}(r) .
$$

If $\mathfrak{x}^{\prime}=\mathfrak{x}^{\prime \prime}$ then, by a suitable application of the isoperimetric inequality, even $D\left[\mathfrak{x} ; u_{0},\right] \leqq l^{2}(r) / 4 \pi$. Both conclusions contradict the assumption $r \Phi^{\prime}(r)<2 \beta_{1} \Phi(r)$. Therefore, indeed, $2 \beta_{1} \Phi(r) \leqq r \Phi^{\prime}(r)$ for almost all $r$. Integration between the limits $r=v_{0}+\rho$ and $r=v_{0}+\delta$ gives

$$
\begin{aligned}
D\left[\mathfrak{x} ; w_{0}, \rho\right] & \leqq D\left[\mathfrak{x} ; u_{0}, v_{0}+\rho\right]=\Phi\left(v_{0}+\rho\right) \leqq\left(\frac{v_{0}+\rho}{v_{0}+\delta}\right)^{2 \beta_{1}} \Phi\left(v_{0}+\delta\right) \\
& \leqq\left(\frac{2 \rho}{\delta}\right)^{2 \beta_{1}} D_{P}[\mathfrak{x}]=M \rho^{2 \beta_{1}},
\end{aligned}
$$

where $M=(2 / \delta)^{2 \beta_{1}} D_{P}[\mathfrak{x}]$. The inequality is proved. 
Statements about the end points of the trace are possible if the Jordan arc $\Gamma$ meets the surface $T$ properly. $\Gamma$ is said to satisfy a LO (=lift-off) condition with constant $c_{2}>0$ if there is a constant $\tau_{0}, 0<\tau_{0} \leqq 1 / 2$, such that

$$
\begin{array}{ll}
\int_{0}^{\tau}\left|d_{z}\right| \leqq\left(1+c_{2}\right) d_{T}[z(\tau)] & \text { for } 0<\tau \leqq \tau_{0}, \\
\int_{\tau}^{\pi}\left|d_{z}\right| \leqq\left(1+c_{2}\right) d_{T}[z(\tau)] & \text { for } \pi-\tau_{0} \leqq \tau<\pi .
\end{array}
$$

If $\Gamma$ is a regular curve of class $C^{1}$ issuing from $T$ (here assumed to be of class $C^{1}$ ) at angles not smaller than $\alpha>0$, then we can set $c_{2}=\epsilon$ $+(1-\sin \alpha) / \sin \alpha$ for arbitrary $\epsilon>0$.

THEOREM 3. If the bounding surface $T$ satisfies a $C A$-condition with constant $c_{1}$ and if the arc $\Gamma$ satisfies an LO-condition with constant $c_{2}$, then the trace-image of the closed arc $\partial^{\prime \prime} P$-belongs to class $C^{0, \beta_{2}}$; i.e. the vector $\mathfrak{x}(u, 0)$ belongs for $-1 \leqq u \leqq 1$ to $C^{0, \beta_{2}}$. Here $\beta_{2}=0<\beta_{2}$ $<2\left(2+c_{1}\right)^{-2}\left(2+c_{2}\right)^{-2}$.

Using well-known facts about minimal surfaces (see $[13$, p. 238]) we conclude

THEOREM 4. If, in addition to the assumptions of Theorem 3, the Jordan arc $\Gamma$ satisfies a $C A$-condition with constant $c_{3}$, then the vector $\mathfrak{r}(u, v)$ is of class $C^{0, \beta_{3}}(\bar{P})$. Here $\beta_{3}=\min \left(2\left(2+c_{3}\right)^{-2}, 2\left(2+c_{1}\right)^{-2}\left(2+c_{2}\right)^{-2}\right)$.

Theorem $4^{\prime}$. If $T$ is a surface of class $C^{1}$ as above and if $\Gamma$ is a regular curve of class $C^{1}$ issuing from $T$ at angles not smaller than $\alpha>0$, then the vector $\mathfrak{r}(u, v)$ belongs to class $C^{0, \gamma}(\bar{P})$. Here $\gamma$ is any number in the interval

$$
0<\gamma<\frac{1}{2} \sin ^{2} \alpha(1+\sin \alpha)^{-2} .
$$

Weaker statements are possible if $\Gamma$ is tangent to $T$ at its end points.

ACKNOWLEDGMENT. The preceding research was sponsored by the Air Force Office of Scientific Research under AFOSR Grant No. 883-67.

\section{BIBLIOGRAPHY}

1. R. Courant, The existence of a minimal surface of least area bounded by prescribed Jordan arcs and prescribed surfaces, Proc. Nat. Acad. Sci. U.S.A. 24 (1938), 97-101.

2. - The existence of minimal surfaces of given topological structure under prescribed boundary conditions, Acta Math. 72 (1940), 51-98. MR 2, 61.

3. - On Plateau's problem with free boundaries, Proc. Nat. Acad. Sci. U.S.A. 31 (1945), 242-246. MR 7, 68. 
4. - Dirichlet's principle, conformal mapping, and minimal surfaces, Interscience, New York, 1953. MR 12, 90.

5. R. Courant and N. Davids, Minimal surfaces spanning closed manifolds, Proc. Nat. Acad. Sci. U.S.A. 26 (1940), 194-199. MR 1, 244.

6. N. Davids, Minimal surfaces spanning closed manifolds and having prescribed topological position, Amer J. Math. 64 (1942), 348-362. MR 3, 250.

7. S. Hildebrandt, Über Minimalflüchen mitfreiem Rand, Math. Z. 95 (1967), 1-19. MR 34 \#8287.

8. W. Jäger, Behavior of minimal surfaces with free boundaries, Comm. Pure. Appl. Math. (to appear).

9. H. Lebesgue, Sur le problème de Dirichlet, Rend. Circ. Mat. Palermo 24 (1907), 371-402.

10. H. Lewy, On minimal surfaces with partially free boundary, Comm. Pure. Appl. Math. 4 (1951), 1-13. MR 14, 662.

11. E. J. McShane, Parametrization of saddle surfaces with applications to the problem of Plateau, Trans. Amer. Math. Soc. 35 (1933), 716-733.

12. C. B. Morrey, On the solutions of quasi-linear elliptic partial differential equations, Trans. Amer. Math. Soc. 43 (1938), 126-166.

13. J. C. C. Nitsche, On new results in the theory of minimal surfaces, Bull. Amer. Math. Soc. 71 (1965), 195-270. MR 30 \#4200.

14. - The behavior of minimal surfaces with free boundaries, Rend. Circ. Mat. Palermo (to appear).

15. I. F. Ritter, Solution of Schwarz' problem concerning minimal surfaces, Univ. Nac. Tucumán Rev. Ser. A 1 (1940), 40-62. MR 3, 55.

University of Minnesota, Minneapolis, Minnesota 55455 